\title{
Ritmos Diatónicos - isomorfismos entre los patrones rítmicos y de alturas, inaturalidad o arbitrariedad?
}

Antenor Ferreira Corrêa

(UNB)

Edwin Pitre-Vásquez

(UFPR)

Resumen: Esta investigación tiene como objetivo verificar las posibles determinantes que conducen a escoger patrones rítmicos característicos. Estos patrones rítmicos forman y diferencian los diversos géneros musicales de las diferentes culturas. Partimos de la hipótesis que la constitución de un patrón rítmico, no es un proceso aleatorio, y si resultante de la junción entre lo que se escoge como más expresivo de los valores del grupo que las aplica, asociado a un factor condicionante: el grado de complejidad de ese grupo. Los resultados, todavía iniciales, ya indican que el proceso de génesis de los géneros musicales no es natural, pero también no es del todo arbitrario, una vez que presenta la existencia de orientaciones funcionales en el empleo de los componentes estructurales del lenguaje musical.

Palabras-Ilave: ritmos diatónicos, configuraciones de las escalas, isomorfismos, timeline patterns.

DIATONIC RHYTHMS - ISOMORPHISMS BETWEEN PATTERNS OF RHYTHM AND PITCH: NATURALNESS OR ARBITRARINESS?

Abstract: This research aims to verify possible determinants that lead to choose characteristic rhythmic standardizations. Rhythmic patterns form and differentiate the various musical genres from different cultures. We hypothesized that a rhythmic pattern formation is not a random process, and if it results from the junction between what is chosen as the most expressive of the values of the group that applies this plus a conditioning factor: the group's complexity degree. In spite of the results earliness, they indicate that the musical genres genesis process is not natural, but nevertheless it is not entirely arbitrary, once it presents the existence of functional guidance on the use of the musical language structural components.

Keywords: diatonic rhythms, scales settings, isomorphisms, time-line patterns.

\footnotetext{
1 Isomorfismo: es un concepto que proviene de la matemática donde dos estructuras poseen la misma forma, es decir un sistema de comparación. Aqui utilizado como esta caracteristica comparativa en los ritmos diatónicos.
} 


\section{Introducción - Frases Rítmicas de Referencia}

En una rápida sintonizada por las estaciones de radio podemos entrar en contacto con algunos géneros musicales actualmente de moda. Encontramos, por ejemplo, manifestaciones musicales como: Rock, RAP, Pop, Funk, Reggae, Salsa, Samba. Estos y otros géneros musicales existentes y se diferencian por presentar estructuras rítmicas características que asociadas al texto, instrumentación y a los elementos externos de la música - como, por ejemplo, vestuario, hablar común, actitud, postura y demás hábitos - componen y permiten identificar el grupo practicante o seguidores del estilo en cuestión. Dentro de una misma categoría estilística pueden existir subdivisiones. La samba, por ejemplo, presenta diversas derivaciones, como samba-canção, samba-de-breque, samba-de-roda, sambaenredo, partido alto y bossa nova, entre otros. A pesar de sus especificidades, todas esas variantes se encuadran en una categoría común: la samba. ${ }^{2}$

Se considera la samba de partido-alto, originario do Rio de Janeiro. Donde la comunidad de "practicantes" posee una manera propia de hablar (compuesta de modismos y expresiones del 'malandro carioca'), de comportamiento y de vestirse. La samba de partido-alto posee también un patrón rítmico característico (Ejemplo 1) que lo difiere de los demás toques de samba.

Ejemplo 1: patrón rítmico característico de la samba de partido-alto

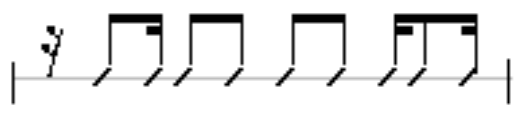

Este patrón rítmico característico recibe comúnmente la denominación de frase rítmica de referencia. Cada género musical posee su propia configuración rítmica patrón, lo que los norte-americanos Ilaman de groove o los brasileños de

2 Pitre-Vásquez propone una clasificación básica para la Samba brasileña. (Pitre-Vásquez, 2000, p. 79). 
levada, que asociada a elementos interpretativos, como articulación y agógica, forman y transmiten la "sensación" (feeling) de cada género. ${ }^{3}$

Gerhard Kubik, en su estudio realizado sobre la música africana, acuño el termo time-line patterns para designar estos tipos de configuraciones patrón. Ese concepto es utilizado aquí, pero con una traducción usual: frases rítmicas de referencia. Segundo Kubik, una frase rítmica de referencia [time-line patterns] "es el corazón estructurador de una pieza musical, algo como la representación condensada y extremamente concentrada de las posibilidades de movimiento y ritmo a disposición de los ejecutantes (músicos o bailarines)" (Kubik, 1981, p. 93). ${ }^{4}$

La pregunta que surge, objeto de este trabajo, es: de donde vienen y como son formadas estas patrones rítmicos? Son creaciones arbitrarias o poseen alguna orientación natural? En una escala o cualquier patrón rítmico resultante de procesos aleatorios 0 es posible encontrar factores determinante en su constitución?

Normalmente, se asume que el origen de un género musical, principalmente cuando se trata de manifestaciones populares, se constituye de un proceso arbitrario. En determinado momento fue "inventado" por alguien, cuya autoría no es posible identificar y aceptado por la comunidad donde surgió, pasando a ser transmitido para las generaciones subsecuentes. El patrón rítmico establecido es, por lo tanto, una de las posibles combinaciones resultantes de diversas probabilidades permitidas por una estructura matriz. En el caso de la

\footnotetext{
3 Para este concepto de "red flexible" utilizado por Oliveira Pinto, podemos encontrar otras terminologías como montuno, swing, pampeo, coimbre (Ibid, 2008, p. 72).

${ }^{4}$ El etnomusicólogo africano J. H. Kwabena Nketia realizo estudios de los patrones rítmicos africanos y desarrollo este sistema de análisis, que es utilizado por otros estudiosos para patrones rítmicos de matrices africanas. El término time-line-pattern ha sido traducido por varios autores latinoamericanos como "líneas temporales" Rolando Pérez Fernández (1986), Maria Helena Vinueza González (1988), "patrones de tiempo asimétricos" o "patrones metrorrítmicos asimétricos" Leonardo Acosta (2004), "linhas-guias" Carlos Sandroni (2001), "frases rítmicas de referência" Antenor Ferreira (2006). Otros sistemas de notación musical para los patrones rítmicos son el Box Notation Method también llamado de 'sistema de cajitas por unidad de tiempo' criado por Philip Harland en 1962 en la UCLA, en 1983 surgió el Time Unit Box System (TUBS), a partir de la utilización de dígitos numéricos. Jeff Pressing crio el sistema conocido como "patrón estándar" y en 1991 Simha Arom crio otro sistema el Rhythmic oddity property. (Ibid, 2008, p. 70).
} 
configuración del ritmo, la matriz es compuesta por las cantidades de tiempos o pulsos, de pulsaciones intrínsecas a ese pulso y de los valores de duración involucrados.

Todavía, proponemos como tesis que en el interior de esta aparente arbitrariedad de génesis de configuraciones rítmicas, existan elementos invariables, o sea, elementos comunes a las varias manifestaciones musicales. Estos elementos son indicadores de que estas creaciones no son tan aleatorias como se podría suponer a primera vista, mas que resultan de un proceso orientado. Esta orientación es funcional y busca la afirmación de los valores de identidad de la comunidad socio-cultural en que ocurren.

Teniendo en vista esas consideraciones, en este trabajo pretendemos levantar puntos de reflexión sobre los modos de configuración de las estructuras musicales rítmicas y escalares, evaluando las variables colocadas en juego durante su constitución. Partimos de la constatación de que el hecho musical, consensualmente entendido como cultural, trae en si elementos universales, como las escalas. A partir de allí, por medio de la transposición del dominio sonoro para el temporal, investigamos la existencia de configuraciones temporales patrón denominadas "ritmos diatónicos". Al examinar los aspectos rítmicos tomamos como base el método de Jeff Pressing (1983) que, en principio, sugiere una posible interferencia de un componente natural en la estructuración rítmica, similar a la que encontramos en la serie armónica referente a las alturas sonoras. Sin embargo, con las investigaciones de Pressing sobre las culturas africanas, tailandesas y de los Balcanes, realizamos aquí la utilización de su modelo en algunos ritmos de la tradición brasileña y afrocubana. Como resultado de este estudio la comprobación de ese modelo, aunque inadecuado para ciertos tipos de pulsaciones subyacentes, apunta para la existencia de un elemento no arbitrario en la constitución de las varias configuraciones analizadas. 


\section{Sobre los orígenes}

Especulaciones al respecto de aquellos que podrían ser los indicios de las primeras manifestaciones musicales conducen, normalmente, el paso del nomadismo para el sedentarismo. Al final de la última Edad del Hielo y con aproximadamente 10,000 a.C., los homo-sapiens, gradualmente abandonan la actividad anterior nómada de colecta y establecen los primeros grupos dedicados al cultivo de la tierra. El cambio de colectores-cazadores para agricultores fijo efectivamente el hombre en locales determinados. Enseguida fueron formadas las primeras comunidades, surgiendo las relaciones sociales y criando nuevas maneras de comunicación. A partir de este momento, las primeras relaciones culturales se intensifican debido a las nuevas condiciones y pre-requisitos necesarios para garantizar la perpetuación de la especie. Además de establecer esa convivencia comunitaria, la estructura agrícola y pastoril proporciono la estratificación social en la medida en que fue necesario, a medio plazo, una organización política responsable por el gerenciamiento y almacenaje del excedente de la producción agrícola. Ese aspecto indica la existencia de individuos disponibles para esa tarea, hecho que también puede sugerir la presencia de otras personas dispensadas del trabajo relacionado directamente al plantío, cuyo tiempo podría ser dedicado a otras tareas, como la metalurgia, la religión o la música.

Suponemos que hubo la constitución de varios grupos formados por otros individuos provenientes de otras regiones o disidentes de la comunidad de origen, nuevos colectivos y poseedores de características propias que los distinguían de los demás. Podemos entender que estas nuevas agrupaciones poseían como materia prima para su creación musical aquello que era dado por la propia naturaleza, o sea, el sonido. El componente cultural surge de la manera como este sonido es tratado por las diferentes comunidades. Las fronteras entre lo natural, un dato físico-acústico, o lo cultural, modos de organización sonora, comienzan a ser unidas desde el momento en que se delinean. Suponemos que las primeras manifestaciones deben haber sido por los artefactos de percusión y deben haber 
sido las primeras formas de ordenamiento sonoro-temporal, sea para auxiliar en los aspectos ritualísticos o apenas como comunicación.

Otras hipótesis, no en tanto, le dan un papel distinto a la música. Rousseau (Cf. 1991) sitúa la inflexiones vocálicas como anteriores a el lenguaje verbal. Según su concepción, las primeras manifestaciones lingüísticas serian compuestas por inflexiones, gritos, vocalizaciones, risas y diversas entonaciones, que varían en altura, duración, timbre, intensidad e indicaban estados afectivos. La convivencia social, entretanto, exigió una expresión precisa y menos apasionada de las ideas, dando lugar al surgimiento a las articulaciones consonantes. Cuando se da el paso de la pasión para la razón, los aspectos melódicos dan lugar a la gramática y, posteriormente, a la argumentación lógica. Las antiguas vocalizaciones e inflexiones melódicas no son del todo abandonadas, más direccionadas para una nueva forma de expresión: la música.

Para el físico Juan Roederer la música formo parte en el proceso evolutivo de la especie humana y obviamente debe tener un valor para la sobrevivencia. Roederer se fundamenta en la llamada "motivación del proceso cognitivo", en el cual para cada percepción sensorial participante del proceso corresponde una respuesta por parte del sistema límbico (motivación), que puede inhibir o modificar la atención perceptiva para los estímulos en cuestión. La percepción musical envuelve tareas cognitivas complejas, desde el instante en que el estímulo acústico es captado por el aparato auditivo hasta su transmisión (envio, análisis, comparación, almacenaje, recuperación) e interpretación por el cerebro. Para Roederer, en este proceso estarían comprendidas las respuestas límbicas biológicamente programadas que serian las "recompensas para perfeccionar nuestra atención auditiva y también su respectiva análisis, almacenaje y vocalización de sonidos, objetivando la adquisición de la lenguaje" (Roederer, 1998, p. 265).

El aspecto comunicativo, en cualquier tipo de lenguaje, reivindica la existencia y el dominio de un código común. Este código, es compuesto de elementos estructurales y por normas de organización, la sintaxis. Así, al mismo tiempo en que comunica una posición individual, el lenguaje expresa todo un 
sistema colectivo y virtual implícito en el código utilizado. En la música, a pesar de un concepto genérico, se entienden por estructuras: escalas, melodía, ritmo, contrapunto, armonía y forma, o sea, el concepto comprende todos los componentes del lenguaje musical, desde las propiedades acústicas básicas hasta las construcciones formales enteras. Esos componentes estructurales son organizados de acuerdo con las reglas de la sintaxis musical. Podríamos indagar si los componentes estructurales se encuentran necesariamente presentes en cualquier tipo de manifestación musical. La respuesta apunta para un dato mínimamente curioso, la ocurrencia de un elemento universal en música.

\section{Elementos esencialmente universales}

En las diversas relaciones culturales del hombre con su medio es posible identificar elementos que están presentes en cualquier grupo social y son llamados "universales". Son ejemplos de universales los hábitos alimentares, para cubrirse de la intemperie, el empleo de utensilios y herramientas, las diversas formas rituales, los diferentes tipos de enlaces de casamiento, entre otros. Los antropólogos verificaran inclusive que "la preocupación estética es una constante en la experiencia humana" (Herskovits, 1963, p. 177.) y se constituye, también, como un elemento universal. La música, echo notoriamente de fundamento cultural y artístico, presenta en su constitución elementos que pueden se incluidos en esta categoría de universal. Las formaciones de escalas son una de sus características, ya que es encontrada en cualquier grupo étnico, un continuo sonoro o manifestación sonora que las identifica de alguna manera.

Evidencias colectadas por etnomusicólogos muestran que ninguna cultura musical dispensa el uso de escalas, indicando que "utilización de las relaciones discretas de altura son esencialmente universales" (Burns \& Ward, 1982, p. 242). La variación se da por las distintas maneras de división de la octava, debido a que la segmentación del continuo sonoro no sigue el mismo patrón en las diferentes culturas. El interior de una octava, por ejemplo, en la música hindú es fraccionado en de 22 intervalos y en la árabe-persa de 15 a 24 intervalos, en contrapartida la 
mayoría de las culturas occidentales adopta la división de la octava en 12 partes iguales.

No obstante, se configuren como construcciones arbitrarias en el interior de una actividad también arbitraria (ya que el hecho musical es un fenómeno cultural), las formaciones escalares se hacen presentes en todas las sociedades musicales. Cual seria la razón para esta ocurrencia universal en medio de un hecho arbitrario? Una posible respuesta es ofrecida por la psico-física aliada a la Teoría de la Información. Según esta teoría, cuando nos enfrentamos a altos niveles informativos, la tendencia humana es de realizar una reducción de esta carga informativa por medio de una codificación en categorías de los elementos que constituyen estés mensajes. Esa segmentación en sub-partes ayudan y facilitan el proceso cognitivo, donde se supone que la existencia de las escalas musicales correspondan a este proceso de subdivisión. Una razón neuropsicologia para este proceso de segmentación y compartir es descrito por Roederer de la siguiente manera:

Es más fácil para el cerebro procesar, identificar y almacenar en su memoria una melodía formada por una secuencia temporal de valores discretos de altura que ostenten una cierta relación entre si, proporcionada de cierta manera por la serie armónica, de que por un patrón temporal de alturas que deslice continuamente para arriba y para abajo por todas las frecuencias posibles, cuyo procesamiento, identificación y almacenaje en la memoria exigirían muchos mas bits de información de que una secuencia discreta (Roederer, 1998, p. 259-260).

El código musical comporta elementos estructurales, como también, sus normas de organización o sea, su sintaxis. A principio se puede pensar que la creación de un sistema como ese es arbitrario. Al adoptar un sistema, un grupo social esta seleccionando lo que es más representativo de sus valores. De esta manera es posible ponderar si el proceso de establecimiento de un código ya no se encontraría direccionado para atender o adecuarse a los valores de la comunidad a donde surge. ¿No seria ese un proceso orientado? ¿Como evaluar la carga de arbitrariedad contenida en ese direccionamiento? Podríamos pensar, entonces, en un dato natural (sonido) manipulado culturalmente, pero de 
antemano, orientado para atender a los intereses de determinado medio social. El próximo paso, dado el confronto entre lo natural y lo arbitrario, seria analizar los elementos estructuradores del lenguaje musical, como por ejemplo, las escalas y los patrones rítmicos.

De una manera resumida, en los parágrafos anteriores especulamos al respecto de el establecimiento de los primeros grupos sociales, cuyas necesidades diversas hicieron con que la materia prima sonora (el sonido) fuera trabajado generando la música y por lo tanto, su código. Ese sumario tuvo por objetivo ponderar sobre cuales serian las condiciones y factores que posibilitaran el surgimiento del lenguaje, en particular el lenguaje de la música. Es necesario aclarar que no se trata de un código escrito o un sistema de representación como lo conocemos hoy, más de un sistema oral que viabiliza la reproducción y posterior decodificación por parte de aquellos cuya manifestación musical fuese destinada.

Debemos resaltar que toda música transmite informaciones, en la medida en que demanda mecanismos cerebrales de percepción (aprensión), análisis, procesamiento (comparación), almacenaje y recuperación de datos, independientemente del contenido musical parece vislumbrar mas los estados afectivos que los informativos. Los tres componentes conocidos "emisor - medio receptor" dentro de la Teoría Clásica de la Comunicación, para el camino de cualquier mensaje requieren de receptor y tres fases: (1) captación de ondas sonoras por el tímpano que las transmite al (2) oído interno, responsable por la clasificación de las vibraciones de acuerdo con la frecuencia y las convierte en impulsos nerviosos eléctricos que son (3) enviados al cerebro, donde "la información es procesada, presentada como una imagen de detalles auditivos en cierta área del córtex, identificada, almacenada en la memoria y eventualmente transferida para otros centros del cerebro" (Roederer, 1998, p. 19).

Sabemos que la formación de las escalas musicales están presentes en todas las culturas a través de la historia, y se diferencian apenas por el número de divisiones hechas en su extensión. Sin embargo, existen investigaciones realizadas buscando evaluar de que manera se realizan esas distintas divisiones y como son 
establecidas las relaciones entre los intervalos. Habría alguna relación natural de frecuencia en la forma como el sistema auditivo procesa el estímulo sonoro y define los intervalos de una escala? Todo indica que la respuesta es no.

Las divisiones son arbitrarias. Aunque que exista una gran proximidad con la serie harmónica y con el ciclo de las quintas en la construcción de las escalas, no hay como establecer una regla general válida para todos los pueblos y, según el historiador Curt Sachs, "ni siquiera la octava es un intervalo obvio en la música primitiva" (apud Nattiez, 1984, p.233). Sachs cita como ejemplo la ausencia de este intervalo en la música de los Marind-aim de Nueva Guinea, de los pigmeos Batwa del Congo y de los esquimales de Cariboo (idem, p. 234).

Teorías que sitúan la adquisición de el habla en un momento anterior al surgimiento de las manifestaciones musicales en la cadena evolutiva sugieren que las relaciones de frecuencia de la configuración escalar corresponden a las distancias entre los sonidos usados en el lenguaje oral. De acuerdo con esa hipótesis, el mecanismo relacionado para escoger los sonidos presentes en una escala musical es guiado por la percepción teniendo como base las sonoridades del lenguaje oral, por lo tanto que serian consideradas por el cerebro como las 'mas agradables', ya que este recrea un contexto basado en experiencias anteriores. Investigaciones realizadas por Kubik parecen confirmar esa hipótesis, debido a la diversidad de lenguas encontradas en África mantienen una relación directamente proporcional al número de escalas músicas empleadas en las diversas regiones. “Las escalas africanas y el 'género de la música' son, esencialmente, función del carácter de las lenguas africanas que, en su mayoría, son más o menos lenguas tonales, o por lo menos, presuponen un cierto dúo lingüístico para algunas palabras y frases" (Kubik, 1970, p. 12). El mismo autor, también advierte sobre la posibilidad de que un investigador capacitado puede percibir de antemano el sistema musical de determinada etnia a partir de su lenguaje oral. Según el, "las lenguas tonales -heptatonicas- son bastante características por sus intervalos cortos y pequeños desvíos de una especie de centro tonal" (ibid., p. 12). Esa interdependencia entre lengua y sistema escalar parece ser una constante en las varias etnias africanas ya que, a pesar de las grandes distancias entre estas, es 
posible demostrar la semejanza existente entre la música de las diferentes etnias que mantienen una semejanza de lengua: "pueblos de lenguas parecidas tienen música parecida o, en todo caso, un sistema tonal semejante" (ibid, p. 12). Kubik evalúa que: la multiplicidad de características y atributos observables en las músicas del continente africano pueden ser explicadas por esa relación esencial entre las lenguas y los sistemas tonales. Sin embargo, evidencias colectadas por etnomusicólogos demuestra que ninguna cultura musical dispensa el uso de escalas, indicando que "la utilización de relaciones discretas de altura es esencialmente universal" (Burns \& Ward, 1982, p. 242).

Siendo posible entender que una escala y la expresión de los intervalos de la serie armónica condensados en el interior de una octava, podría juzgar los patrones rítmicas como prolongaciones de una propiedades físico-acústica del sonido, como la duración? La escogencia o constitución de un patrón rítmico seria mas arbitraria de que el proceso de formación de las escalas?

\section{Ritmos diatónicos}

Tenemos, por un lado, un dato natural (el sonido) y por otro, la manera de cómo este es estructurado, un hecho cultural. Las alturas la serie armónica, funcionan como una base física en la aproximación de lo natural con lo cultural. En el dominio temporal, no obstante, las sub-divisiones parecen que están revestidas de una carga arbitraria mayor. Todavía, en algunos estudios (en especial Pressing, 1983) procuraron verificar cuan alto seria este grado de arbitrio, trabajos que acabaron por señalar ciertas coincidencias entre las estructuras escalares diatónicas y algunas frases rítmicas de referencia, los denominados "ritmos diatónicos". Un ritmo diatónico es el resultado de una segmentación en el dominio temporal que genera un patrón rítmico cuya configuración interna es similar a la disposición intercalar de una escala o modo diatónico. La descubierta de los ritmos diatónicos llevo en un primer momento, a percibir una especie de componente natural comandando el proceso de constitución de los ritmos, debido a que si entendemos que una formación escalar resulta de procedimientos 
"naturales" y ya que los patrones rítmicos se encuentran, de cierta forma, vinculados a las estructuras internas de las escalas, estos ritmos deben, por consecuencias, poseer también un tipo de determinante natural.

El proceso de transportación entre el dominio escalar para el rítmico funciona de la siguiente manera: los patrones rítmicos primordiales son trasladados para el dominio sonoro por medio de la conversión de sus valores de duración temporal en intervalos de tonos y semitonos. Para esto es necesario examinar inicialmente las configuraciones escalares.

Cada conjunto o secuencia escalar diatónica posee una configuración de tonos y semi-tonos que varia de acuerdo con la disposición interna de los mismos. Examinemos los siguientes conjuntos diatónicos:

Ejemplo 2: Estructuración intervalar de los modos diatónicos

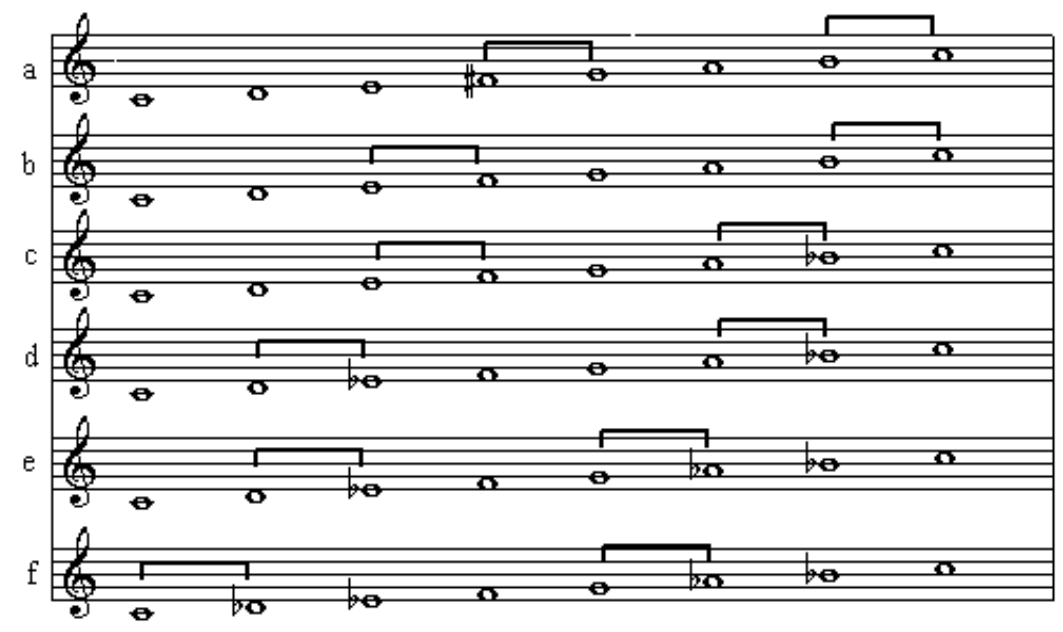

En el Ejemplo 2, es demostrada la relación interna de tonos y semi-tonos correspondientes a los modos diatónicos. Las llaves sobre las notas indican las posiciones de los semi-tonos en cada nueva construcción. Podemos observar que un único conjunto de intervalos (misma cantidad de notas o matriz) conteniendo seis tonos e dos semi-tonos origina distintos patrones diatónicos apenas por el reordenamiento de la configuración interna del conjunto (existen otras posibilidades de disposición para este conjunto de intervalos no demostradas en 
esta figura), siendo que cada nuevo patrón formado posee carácter y cualidades distintas entre si.

Análogamente a esas configuraciones escalares diatónicas, podrían generar patrones secuenciales rítmicos correspondientes de la siguiente manera: (1) Observemos el Ejemplo 3a los patrones correspondientes a las formaciones escalares a y d del Ejemplo 2 (conocidos como modos lidio y dórico, respectivamente), organizados por su disposición de tonos (T) y semitonos (S). (2) Se substituyen, por intervalos de tonos enteros por una negra y los de semi-tonos por una corchea, como mostrado en el Ejemplo $3 \mathrm{~b}$ :

Ejemplo 3: (a) modos lidio y dórico organizados según sus secuencias de tonos y semitonos; (b) respectiva substitución de los tonos y semi-tonos de los modos lidio y dórico por valores de duración.

$\mathrm{a}$

\section{\begin{tabular}{|l|l|l|l|l|l|l|}
\hline $\mathrm{T}$ & $\mathrm{T}$ & $\mathrm{T}$ & $\mathrm{S}$ & $\mathrm{T}$ & $\mathrm{T}$ & $\mathrm{S}$ \\
\hline
\end{tabular}}

\begin{tabular}{|l|l|l|l|l|l|l|}
\hline $\mathrm{T}$ & $\mathrm{S}$ & $\mathrm{T}$ & $\mathrm{T}$ & $\mathrm{T}$ & $\mathrm{S}$ & $\mathrm{T}$ \\
\hline
\end{tabular}
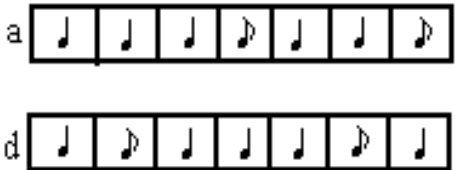

En el Ejemplo 4, son presentadas las estructuras básicas de los ritmos Barra-vento (presente en el candomblé afro-brasileño) y del Bembé (tradición afro-cubana. Cf Malabe, 1990, p. 8). Las figuras correspondientes a los valores de duración fueron convertidas en intervalos de tono (negra) y de semitono (corchea), dando origen a dos modos, o escalas, diatónicas: lidio y jonio, respectivamente. Por esta razón (coincidencia entre los patrones de tiempo y de alturas) estos ritmos fueron llamados de "ritmos diatónicos".

Ejemplo 4: Correspondencia entre las configuraciones de intervalos y rítmicas. Modo lidio y Barra-vento (a) y modo jonio y Bembê (b)

(a)

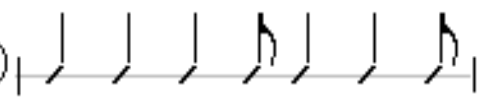

a

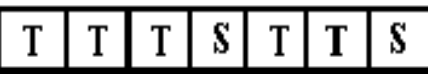

(b)

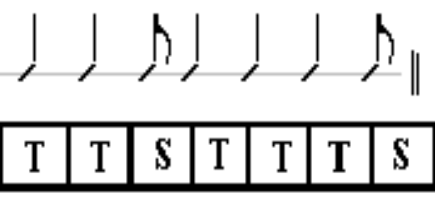


Otras formaciones son posibles, como en el Ejemplo 5. El ritmo cubano Abakwa es transpuesto para el dominio frecuencial resultando en una escala pentatónica (Ex. 5b). De manera similar es posible invertir el proceso, transportándolo al modo lidio con la séptima menor (o mixolidio con cuarta aumentada), modo frecuentemente utilizado en la música tradicional del Noreste del Brasil, y tendrá como resultante el dominio temporal la secuencia rítmica mostrada en el Ex. 5 a.

Ejemplo 5: (a) Transposición de los modo mixolídio con cuarta aumentada (o lídio con séptima menor) para el dominio temporal; (b) Patrón básico del Abakwa transportado para el dominio de las alturas resultando en una escala pentatónica.

(a)

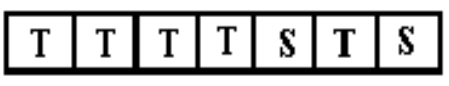

(a)

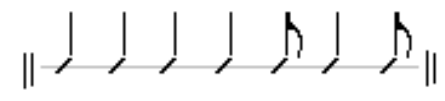

(b)

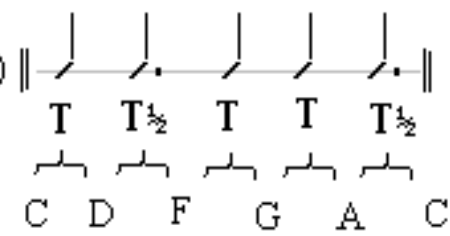

Transposiciones semejantes pueden obtenerse en cualquier ritmo, pero solamente aquel cuyo grupo métrico es de doce o de seis corcheas (fórmula del compás $12 / 8$ o 6/8) resultara similar a los modos diatónicos. Vale la pena notar que la estructura de los patrones rítmicos formados en la transposición entre dominios corresponde al agrupamiento métrico de doce pulsaciones, que pueden ser escritas en compás cuaternario compuesto teniendo como unidad de tiempo la negra con puntillo (formula de compás 12/8), grupo rítmico típicamente característico y significativo de músicas tradicionales africanas, como resalta Kubik:

Las formas musicales (africanas) se organizan de forma que los motivos y temas se desarrollan de acuerdo con un número regular de esas pulsaciones elementares, habitualmente $8.12,16,24$ o sus múltiplos, pero raramente 9 , 18 o 27 pulsaciones. Es a lo que Ilamamos de ciclos; los números son designados por formulas estructurantes. Muchas de estas fórmulas pueden ser dividas o partidas de varias maneras, permiten la combinación simultanea de unidades métricas contradictorias. Por ejemplo, el número 12 , que es el más importante en la música africana, puede ser dividido por 2, 3 , 4 e 6. (Kubik, 1981, p. 92). 
No obstante, esas correspondencias isomorfas son apenas coincidencias, que no presentan cualquier sustentación cuando se especula al respecto de determinaciones naturales. Esa ausencia de base natural puede ser analizada si se observa, que solamente para los grupos métricos cuya pulsación intrínseca es de doce o de seis corcheas (compás de 12/8 o 6/8) los patrones rítmicos resultaran similares a los modos diatónicos. Utilizando cualquier ritmo cuya pulsación subyacente resulte diferente de 12 o 6, como los ritmos de 16 pulsaciones mostrados en el Ejemplo 7a e 7b, la escala que se obtiene no será diatónica. Las escalas formadas en el Ejemplo 7 poseen nueve intervalos, quiere decir, que sobre pasan la tesitura de una octava, generando una escala sintética (cf. Persichetti, 1961, p. 41) por presentar desvíos en la secuencia normal de los sonidos parciales superiores originados en la serie armónica.

El Ejemplo 6a muestra una de las frases frecuentes de la samba, como también su pulsación subyacente en semicorcheas, que trata de la fórmula simples de compás. En el Ejemplo 6b, no obstante la pulsación subyacente es de corcheas, pues se trata de un compás compuesto. Notamos que el modo formado en $6 \mathrm{~b}$ es un modo diatónico conocido: el dórico. Todavía, en el Ejemplo $6^{\mathrm{a}}$ la configuración que resulta posee nueve intervalos, criando así, una escala sintética. En el Ejemplo 7, se demuestran las configuraciones de intervalos en las escalas sintéticas resultante de dos ritmos tradicionales brasileños: el Maracatu y el ljexá; Ambos poseen pulsaciones subyacentes de semicorcheas.

Ejemplo 6: (a) Patrón rítmico de la Samba y (b) configuración resultante del modo dórico
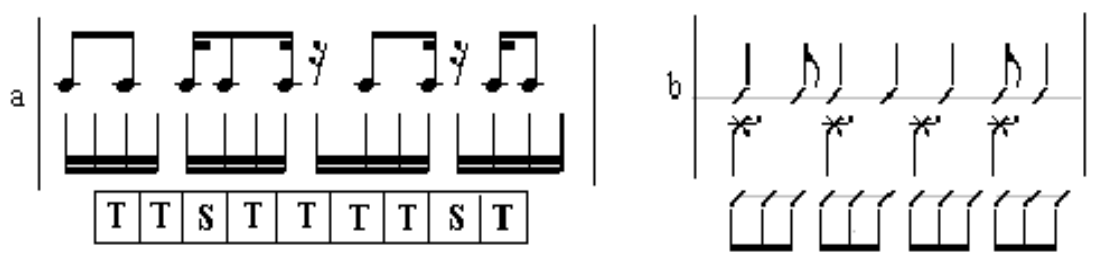
Ejemplo 7: frases rítmicas del Maracatu (a) y del ljexá (b) con sus respectivas transposiciones para el dominio entre intervalos.

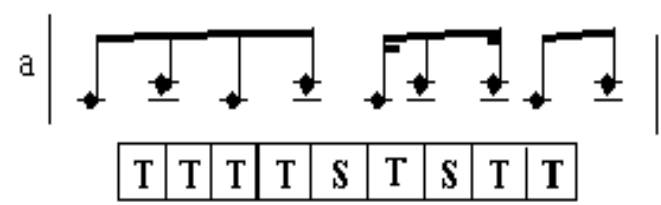

b
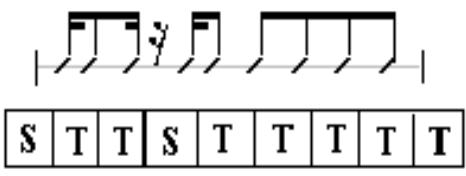

\section{Conclusiones}

Los modos de relación del hombre con su medio, como también la aceptación y transmisión de estos modos, entre otras cosas, están comprendidos en la cultura. Lo que se perpetúa y se convierte en tradición, es aquello que es importante para el grupo social. Los legados solo se mantienen por la preservación de valores que vienen implícitos, valores estos que en un dado momento atribuyeron y constituyeron la identidad de la comunidad a donde surgieron. Por lo tanto, es posible especular que el proceso de creación o de surgimiento de elementos culturales sea norteado por el principio de la identidad cultural. Entre las varias posibilidades lo que se impone y se establece es algo que identifica el grupo. La expresión de los valores significativos es intrínseca al proceso de creación. Yuxtapuesto a esto, se observa el aspecto de poder distinguir entre los grupos sociales. Normalmente las manifestaciones culturales sirven como diferenciales entre las comunidades, por consiguiente, se supone que el grupo "elija" como representativo aquello que no sea semejante a expresiones de otros grupos. Hecho que puede ser notado en el relato de Kubik:

Regiones muy distantes entre si presentan con frecuencia trazos similares o mismo idénticos, en cuanto áreas contiguas pueden presentar diferencias estilísticas. La música polifónica de los Baulé, de la Costa de Marfil, a tres voces y en el sistema tonal equi-heptatónico esta bien próximo, podemos decir que es idéntica, de la música vocal de los Ngangela, Chokwe y pueblos de lengua Luvale del Este de Angola, y como tal reconocida por informantes de varias culturas referidas. Las dos regiones están separadas por varios países con formas muy diferentes de canto polifónico (1991, p. 91). 
De esta manera, entendemos que aspectos como la transculturación, la influencia y sincretismo sean pasos posteriores de este proceso.

Comprendido entre estos dos extremos -identidad y distinciónencontramos la problemática de la complejidad. No se entiende la complejidad sobre el punto de vista evolutivo-positivista, en que adquiere un estatuto valorativo, mas si como un proceso entrópico, que se obtiene por el aumento de elementos y/o incremento de los aspectos involucrados en la convivencia sociocultural. Es erróneo postular que la música de determinado pueblo se encuentra en una etapa inferior por que su canto es unísono, comprendido en una gama pentatónica con pulso rítmico apenas en el tiempo fuerte. Entendemos que el pulso es el elemento básico de la estructuración musical, cualquier elaboración realizada sobre él, implica consecuentemente, en el aumento de la carga informativa y de la complejidad.

Por cuenta de esto, resaltamos que el grado de complejidad en que se encontraba determinada cultura en un determinado momento, afecta la constitución de la música y de los patrones rítmicos. Postulamos entonces, que las manifestaciones culturales reflejaban los valores representativos y diferenciales de la comunidad de donde surgieron, elementos estos que yuxtapuestos al grado de complejidad en que se encontraba el grupo en aquel momento, actúan en la elaboración musical y en la conformación de las configuraciones rítmicas.

A pesar de la multiplicidad de funciones y atributos presentados por el hecho musical, indicando para la imposibilidad de la existencia de una música universal, algunas propiedades de este hecho pueden ser consideradas de uso común por la gran mayoría de los pueblos, como es el caso de las escalas. La segmentación del continuo sonoro en unidades discretas es descrita por etnomusicólogos como "esencialmente universal". La necesidad de división del campo de las alturas puede tener como objetivo la reducción de la carga informativa presentada a los individuos de manera a auxiliar el proceso cognitivo, facilitando el procesamiento cerebral. De manera análoga, el continuo temporal presenta subdivisiones peculiares, lo que llevo a especular al respecto de la existencia de una especie de transposición entre los dominios sonoros y temporal, 
visualizando una posible orientación natural. Investigaciones realizada en diferentes culturas demuestran la presencia de patrones rítmicos correspondientes a los patrones escalares, llamados de "ritmo diatónicos". En las secuencias de la tradición africana se observo que la construcción de los patrones de referencia presentan en su conjunto 12 elementos (resultante del agrupamiento métrico cuaternario compuesto), semejante a las 12 clases de alturas existentes en las formaciones escalares. En los ritmos como los de la tradición brasileña, se observa que el conjunto es formado por 16 elementos (correspondientes a la pulsación subyacente al patrón general), lo que engendra configuraciones no diatónicas. No obstante, los elementos esencialmente universales existen de hecho, donde se podría especular sobre la necesidad como ocurren.

Rescatando la discusión inicial sobre el código de comunicación musical e su analogía con el código verbal nos permitimos decir que el aspecto comunicativo, en cualquier tipo de lenguaje, reivindica la existencia y el dominio de un código común. Ese código, es compuesto de elementos estructurales y por normas de organización, la sintaxis. La constitución de un sistema de códigos no ocurre de una manera aleatoria o completamente imprevisible.

Como sistema cerrado, se forma a partir de elementos estructurantes, los cuales viabilizan su construcción y determinan sus transformaciones, además de asegurar su coherencia. De las transformaciones concebibles, solamente son realizadas aquellas para las cuales el sistema se encuentra orientado en función de sus estructuras constituyentes. Todos los objetos estables, componentes del sistema, se componen de materia y forma, de significante (trazos visibles y observables, su morfología empírica) y significado (concepto racional que le asegura la estabilidad), de lo perceptible y de lo inteligible, trayendo implícitos un dinamismo subyacente que orienta y determina la organización de la estructura general. Esas directrices intrínsecas serian gobernadas por las estructuras, por ejemplo, la edificación de los códigos comunicativos.

De esa manera, los elementos esencialmente universales ocurrirían por que existen orientaciones necesarias de antemano implícitas en el propio proceso de construcción del sistema general que los abarca. En la música, por ejemplo, la 
necesidad de segmentación de los continuos frecuencial y temporal es una directriz intrínseca a la estructura del código musical, sin el cual, la comunicación no lograría éxito.

De una forma sucinta entendemos que en la música el elemento natural es el sonido con sus propiedades físico-acústicas implícitas. Lo cultural se refiere a las limitaciones e imposiciones del medio, o sea el grado de complejidad de determinado grupo cultural en un momento dado y el deseo de expresión de los valores de identidad de este grupo (lo que se perpetua, y se convierte en tradición, es aquello importante para el grupo social. Los legados solo se mantienen por la preservación de valores que vienen implícitos, valores estos que en un dado momento atribuyeron y constituyeron la identidad de la comunidad a donde surgieron). Arbitrarios son los modos de configuración de materia-prima musical, el sonido. Todavía, como fue posible percibir, esa arbitrariedad no es del todo aleatoria, pues posee ciertas determinaciones u orientaciones impuestas por la propia constitución interna del sistema, característica esa resultante de las estructuras integrantes del sistema general. Ese aspecto no nos permite hablar en las diferenciaciones absolutamente exactas entre lo cultural o lo natural, ya que es un dato físico natural y es de hecho manipulado culturalmente, no obstante esta aplicación sea cercada por orientaciones o pre-disposiciones inherentes a la propia naturaleza de las estructuras integrantes.

\section{Referencias}

BURNS, Edward M. \& WARD, W. Dixon. "Intervals, Scales and Tuning". In: DEUTSCH, Diana (ed.). The Psychology of Music. San Diego: Academic Press, 1982, p. 241-269.

HERSKOVITS, Melville J. Antropologia Cultural. Trad. Maria José de Carvalho e Hélio Bichels. São Paulo: Mestre Jou, 1963.

KUBIK, Gerhard, “African Music”. In: Cultural Atlas of Africa. Oxford: 1981, p. 9093.

. "Natureza e estrutura de escalas musicais africanas". Trad. João Branco. In: Estudos de antropologia cultural. No 3. Lisboa: Junta de Invest. do Ultramar, 1970. 
MALABE, Frank and WEINER, Bob. Afro-Cuban Rhythms for Drumset. New York: Manhattan Music Inc., 1990.

MERRIAN, Alan P. The Anthropology of Music. Evanston: Northwestern University, $7^{\mathrm{a}}$ ed., 1978.

MUKUNA, Kazadi wa. Contribuição Bantu na Música Popular Brasileira: perspectivas etnomusicológicas. São Paulo: Terceira Margem, 2000.

NATTIEZ, Jean Jacques. "Escala" in: Enciclopédia Einaudi. Lisboa: Imprensa Nacional-Casa da Moeda, 1984. Volume 3, p. 229-244.

OLIVEIRA PINTO, Tiago de. "As cores do som: estruturas sonoras e concepção estética na música afro-brasileira". In África: Revista do Centro de Estudos Africanos 22-23 (1) 1999/ 2000/ 2001. São Paulo: FFLCH-USP, 2004.

PÉREZ-FERNÁNDEZ, Rolando. La binarización de los ritmos ternarios africanos en

América Latina. La Habana: Casa de las Américas. 1986.

PERSICHETTI, Vincent. Twentieth Century Harmony - Creative Aspects and Practice. New York: W. W. Norton \& Company, 1961.

PITRE-VÁSQUEZ, Edwin Ricardo. "Clave, Guiro y Maraca" en "Encyclopaedia of Popular Music of the World' editado por John Shepherd et al. Londres: Cassell (no prelo).

"A música na formação da identidade na América Latina: o universo afrobrasileiro e afro-cubano". Dissertação de Mestrado defendida no PROLAM-USP da Universidade de São Paulo, Orientadora: Profa. Dra. Dilma de Melo Silva e Coorientador: Kazadi wa Mukuna, Ph.D, 2000.

"Veredas Sonoras Da Cúmbia Panamenha: Estilos e Mudanças de Paradigma". Tese de Doutorado defendida na Escola de Comunicações e Artes da Universidade de São Paulo. Orientador: Prof. Dr. Eduardo Seincman, 151 pags. 2008.

PRESSING, Jeffrey. "Cognitive Isomorphismos Between Pitch and Rhythm in World Music- West Africa, the Balkans, Thailand and Western Tonality". Studies in Music, Melbourne, v. 17, p. 38-61, 1983.

ROEDERER, Juan. Introdução à Física e Psicofísica da Música. Trad. Alberto Luis da Cunha. São Paulo: Edusp, 1998.

ROUSSEAU, Jean-Jacques. "Ensaio sobre a origem das línguas". In: Coleção Os Pensadores. Tradução Lourde Santos Machado. São Paulo: Nova Cultural, 1991.

SANDRONI, Carlos. Feitiço Decente: Transformação do samba no Rio de Janeiro (1917-1933). Rio de Janeiro: Jorge Zahar 2001. 